\title{
A NOVEL APPROACH TOWARD RAPID ROAD MAPPING BASED ON BEAMLET TRANSFORM
}

\author{
Moslem Ouled Sghaier, Idrissa Coulibaly, Richard Lepage \\ École de technologie supérieure, Montréal, Québec, Canada \\ E-mail : moslem@livia.etsmtl.ca
}

\begin{abstract}
In this paper, a new method for road extraction from very high resolution satellite images is introduced. The proposed methodology is based on multiscale analysis of the image through beamlet transform that can approximate edges of an image by means of line segments appearing at different resolution scales. The hypothesis of the membership of these segments to a road is verified by identification of parallel beamlets representing the main axes of the road. A reconstruction step of the road network is finally applied by aggregation of beamlets representing road candidates belonging to lower scales.
\end{abstract}

Index Terms - Road extraction, Beamlet transform, Multiscale analysis, Haiti earthquake.

\section{INTRODUCTION}

Since the last few decades, the interest towards the field of remote sensing has been increasing. This interest appears in the huge number of satellites orbiting around the earth. The sensors on these satellites provide daily images over regions of the earth for various purposes: mapping, updating geographic databases and change detection. Among the various application fields, we focus on disaster management in which remote sensing contributes to the improvement of the rescue teams response. When a disaster occurs, remote sensing is often the only way to see what is happening on the ground. It is very useful to know if roads are passable or not in order to direct rescue teams on the ground. This paper focuses on the first step of the process dedicated to damage assessment of road network that consists in the extraction of roads from images before and after the disaster. Actually the performance of the damage assessment algorithm is highly dependent on the performance of the method used for road extraction. The remainder of the paper is organized as follows. The second section will present a literature review of road extraction approaches. In section 3 , we briefly introduce the main concept of the beamlet transform. Then, section 4 will be devoted to the presentation of the methodology adopted for solving the problem of road extraction. Finally, we conclude by experimentation and conclusion.

\section{RELATED WORK}

Road extraction approaches are numerous and varied according to the application field, the spatial neighborhood and the input data used. In this literature review, we will adopt the decomposition proposed by Hemiari in [1] who divides roads extraction approaches into local and global approaches. The foundation of local approaches is based on the analysis of the spectral information contained in the current pixel in order to distinguish those belonging to the road. We mention in this context the work of Mohammadzadeh et al [2] in which mathematical morphology has been applied for road extraction: First, the image is classified by fuzzy logic rules, followed by a refinement step resulting from applying mathematical morphology. In [3], texture measurements are used to segment the image and extract roads, the resulting images are then fused using the evidence theory. The limits of those works lie in the treatment of local information only and generation of misclassified pixels. The second family of approach is defined as methods interested in the interpretation of pixel clusters in the image. In [4], the Hough transform is used to extract roads: first the Hough transform is performed to identify linear structures, then a correction step is applied to distinguish roads from buildings using a region growing algorithm. An object-oriented classification is applied in [5] to enhance pixel classification result and to identify roads in the urban scene. The use of object-oriented classification method might overcome the limitation of pixel classification however the choice of parameters remains a challenge and can produce an under- or over-segmentation.

Some research works combine local and global approaches. These approaches are based on multiscale analysis of the image in the identification of the most discriminating scale to extract roads. A multiscale segmentation based on wavelet transform has been described in [6]. The proposed methodology focuses on extraction of the most appropriate scale to describe objects in the image. Roads are subsequently identified by measuring the area to perimeter ratio. Another work was presented in [7], in which extraction of roads from the radar images was performed by applying multiscale analysis based on the beamlet transform. The obtained results are promising.

(C) 2014 IEEE. Personal use of this material is permitted. Permission from IEEE must be obtained for all other uses, in any current or future media, including reprinting/republishing this material for advertising or promotional purposes, creating new collective works, for resale or redistribution to servers or lists, or reuse of any copyrighted component of this work in other works.

The full publication is available at http://dx.doi.org/10.1109/IGARSS.2014.6946943 


\section{BEAMLET TRANSFORM}

The beamlet transform is a multiscale transformation able to represent edges of an image as a collection of line segments (the so-called beamlets) belonging to different levels of resolution. The figure 1 illustrates the beamlet decomposition of an image:
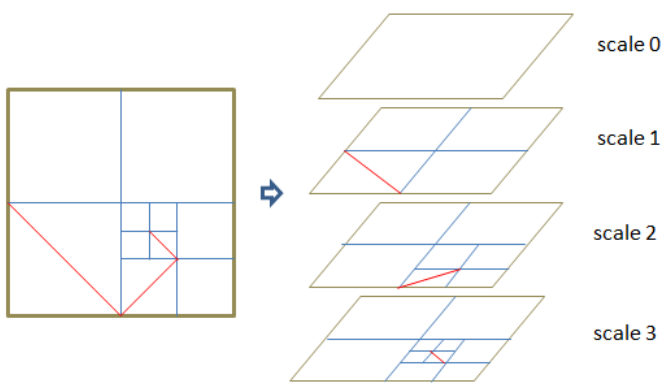

Fig. 1. Beamlets decomposition of an image

\subsection{Case of a continuous function :}

The beamlet transform of a continuous function $f\left(x_{1}, x_{2}\right)$ on $[0,1]^{2}$ is defined in the work of Donoho et al [8] as the collection of all line integrals given by the following formula :

$$
T_{f}(b)=\int_{b} f(x(l)) d l
$$

This equation explains that the application of the beamlet transform of a continuous function involves computing the integral along all line segments.

\subsection{Case of a discrete function :}

By analogy to the continuous case, the digital beamlet transform of an $n \times n$ matrix $\left(f_{i 1, i 2}\right)$ is defined as the beamlet transform of the function $f$ defined on the continuum by interpolation of the values $\left(f_{i 1, i 2}\right)$. Equation 1 is written in the discrete case as follows:

$$
f\left(x_{1}, x_{2}\right)=\sum_{i_{1}, i_{2}} f_{i_{1}, i_{2}} \Phi_{i_{1}, i_{2}}\left(x_{1}, x_{2}\right)
$$

With $\Phi_{i_{1}, i_{2}}$ an interpolation function that allows continuous sampling of the function $f$ to obtain a set of grayscale values.

\subsection{Energy:}

We also associate to each beamlet an energy coefficient defined by:

$$
C_{s}=\frac{T_{f}(b)}{\sqrt{l(b)}}
$$

With $T_{f}(b)$ the line integral of beamlet transform and $\sqrt{l(b)}$ the Euclidean distance between two vertices $v_{1}$ and $v_{2}$ defining the ends of the beamlet $b$.

\section{ROAD EXTRACTION USING BEAMLET TRANSFORM}

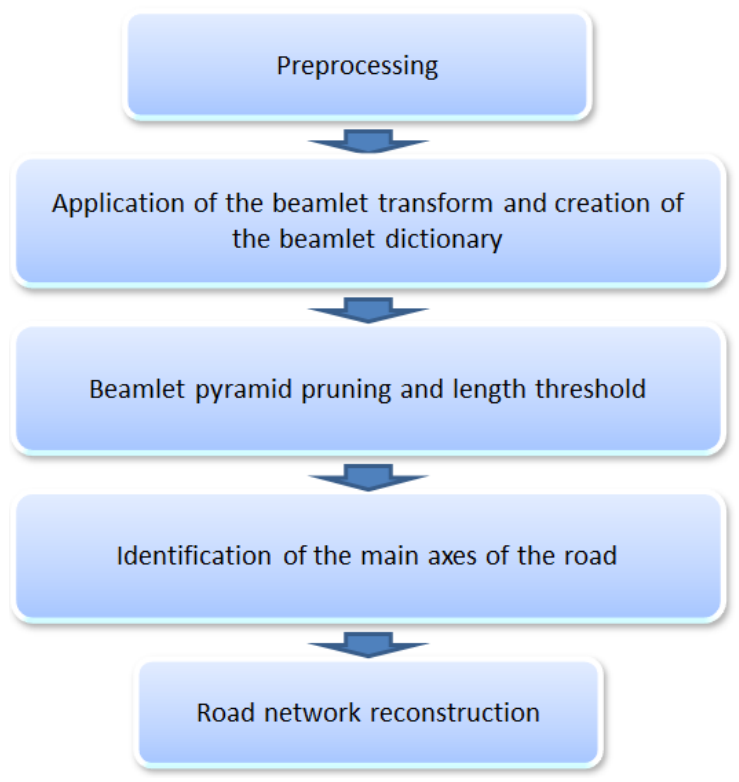

Fig. 2. Global scheme of the proposed methodology

The adopted methodology described in the figure 2 includes five phases: a preprocessing step is first used to compute the edge map of the region of interest by applying the optimal edge detector proposed by Canny in [9]. The second step is based on the application of the beamlet transform to the edge map in order to approximate edges present in the image by means of beamlets from different resolution scales. Then a beamlet pyramid pruning step is applied in order to identify relevant beamlets and eliminate those due to the noise in the image. Segments belonging to roads are then identified by clustering of mutually parallel beamlets. Roads are distinguished from buildings by their length strictly greater than their widths. Thus the calculation of the ratio length by width gives an idea of the membership of a couple of beamlets to a road. Finally, the road network is reconstructed by aggregating beamlets belonging to smaller scales and using beamlet candidates found in the previous phase as seeds for the reconstruction of the road network.

\subsection{Preprocessing}

The purpose of this step is to prepare the image to the road extraction algorithm. Satellite images are generally very large, so it is important in this step to divide the image into subimages in order to facilitate their loading into memory. As the application of the beamlet transform on an image includes the decomposition of the image into dyadic blocks therefore the subimage size have to be a power of 2 . In this work, we set the size of the generated subimages as 512 by 512 . The Canny edge detector was then applied on the image to gener- 
ate an edge map. For the Canny algorithm, we use a Gaussian filter with $\sigma=\sqrt{2}$.

\subsection{Application of the beamlet transform}

Our aim is to extract linear structures present in the image To achieve this objective we perform a mutliscale decomposition of the image using the beamlet pyramid structure. At each level of the pyramid, we look at each dyadic block for the corresponding beamlets. Selected candidates correspond to segments in the image with an energy greater than 0.7 . This threshold was defined experimentally giving a better estimate of the linearity of a segment in the image.

\subsection{Beamlet pyramide pruning}
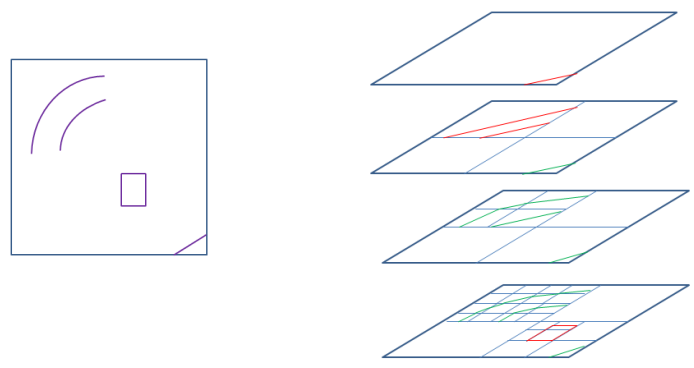

Fig. 3. Application of the pruning step

Applying beamlet transform generates beamlets representing segments of the image, but also produces redundant beamlets with respect to their representation at higher levels. To remedy this problem, we will consider only the first appearance of beamlets in coarser scales. Figure 3 illustrates the application of the pruning step, with the red color indicating the first occurrence of a beamlet while the green color distinguishes beamlets appeared at lower scales.

Segments generated by this phase are not all candidates of the road class since we can also keep: small objects, like noise, in the image. Hence the importance of applying a threshold able to filter irrelevant segments. The choice of the threshold value depends on the resolution of the image.

\subsection{Identification of the main axes of the road}

The beamlets selected in the previous step are identified regardless of the spatial relationships between them. Indeed, two adjacent beamlets may belong to two completely separate objects. The first descriptor that can characterize the roads and remove this ambiguity is the parallelism between the main axes of the same road. We only keep beamlets which satisfy the following equation:

$$
\operatorname{angle}\left(\vec{B}_{1}, \vec{B}_{2}\right) \leq \alpha
$$

With $\vec{B}_{1}$ and $\vec{B}_{2}$ are two vectors characterizing the direction of two beamlets $B_{1}$ and $B_{2}$ and $\alpha$ the angle allowed between the two vectors $\vec{B}_{1}$ and $\vec{B}_{2}$ set in this work to $\frac{\pi}{9}$.

The second descriptor is defined as the ratio of length to width of the road and the degree of homogeneity between two given beamlets. To apply these concepts, we define the following parameter which takes into account these two features:

$$
M_{B_{1} B_{2}}=2 R_{B_{1} B_{2}}+H_{B_{1} B_{2}}
$$

With $R_{B_{1} B_{2}}$ a measure of rectangularity determined by the ratio $\frac{\text { length }}{\text { width }}$ and $H_{B_{1} B_{2}}$ the standard deviation of the region defined by the two beamlets and determined by $\frac{1}{\sigma_{B_{1} B_{2}}}$.

\subsection{Road network reconstruction}

Our aggregation algorithm starts from the list of candidate beamlets provided in the previous step and tries to add more beamlets. This is unlike existing tracking methods described in the literature which attempt to gather arbitrary edges. The aggregation of segments in our case will be based on the results of the decomposition of the image to a beamlet pyramid. The algorithm consists in adding at each iteration neighboring beamlets to the candidates beamlets characterized by similar direction and obeying to the following:

$$
\operatorname{angle}\left(\vec{S}_{r e s}, \vec{B}_{1}\right) \leq \beta
$$

With $\vec{S}_{\text {res }}$ and $\vec{B}_{1}$ the two vectors characterizing respectively the direction of the segment resulting $S$ and the beamlet $B_{1}$ and $\beta$ the angle allowed between the two vectors $\vec{S}_{\text {res }}$ and $\vec{B}_{1}$ set in this work at $\frac{\pi}{6}$.

In some cases, we detect the presence of discontinuities in the edge map due to noise in the image or the presence of objects that occlude the construction of the road. A tracking algorithm is used to find from a window centered at the ends of beamlets neighboring beamlets having the same direction. We set the size of the search window to $5 \times 5$ pixels. Figure 4 describes the two possible scenarios:

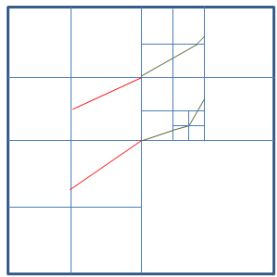

(a)

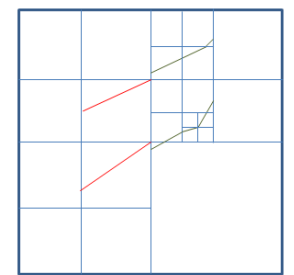

(b)
Fig. 4. Road network reconstruction : (a) Case of connected beamlets (b) Case of disconnected beamlets. 


\section{EXPRIMENTATION}

This work is part of a project which aims at identifying roads and assessing their damage during a major disaster. We are particularly interested in analyzing satellite images acquired before and after the earthquake occurred in Haiti on 12 January 2010. Figures 5 shows two images of the same area taken before and after the disaster.

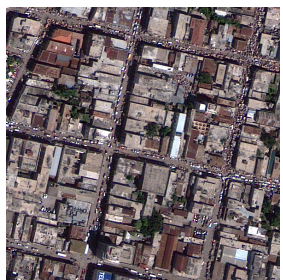

(a)

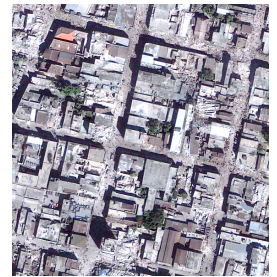

(b)
Fig. 5. City of Port au Prince: (a) Before the disaster (b) After the disaster

The first part of the proposed algorithm for roads extraction has been implemented and the results obtained on a rural area are shown in 6 . We can notice that beamlet transform is able to identify the main axes of the road and approximate them by beamlets. Curvatures present in the structure of the road are represented by beamlets belonging to low resolution levels, which provides relevant information for the reconstruction of the road network.

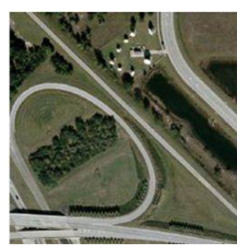

(a)

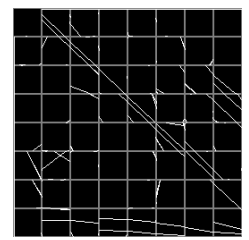

(d)

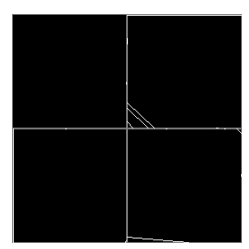

(b)

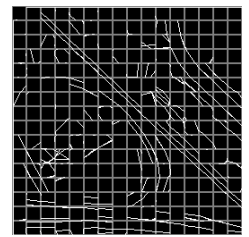

(e)

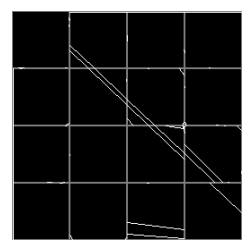

(c)

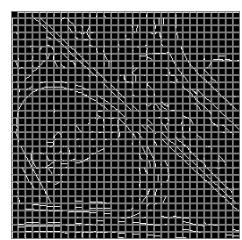

(f)
Fig. 6. Simulation results

\section{CONCLUSION AND FUTUR PROSPECTS}

In this paper, a new algorithm for roads extraction from very high resolution satellite images is presented. The multiscale reasoning based on the beamlet transform was used to extract linear structures in the first step and to distinguish roads in the second step, as well as eliminate small artifacts. The obtained results are promising.

The main perspectives that appear as a result of this work concerns the application of our methodology on satellite images of Haiti, the modification of the proposed algorithm and adapts it to rivers identification. The second perspective will focus on the assessment of roads damage caused by major disasters using multiscale texture analysis.

\section{REFERENCES}

[1] Gholam-Abbas Hemiari, Nouvelle méthode d'extraction automatique de routes dans des images satellitaires, Ph.D. thesis, Sherbrooke University, 2001.

[2] Ali Mohammadzadeh, Ahad Tavakoli, and Mohammad J. Valadan Zoej, "Road extraction based on fuzzy logic and mathematical morphology from pan-sharpened ikonos images," The Photogrammetric Record, vol. 21, no. 113, pp. 44-60, 2006.

[3] J.B. Mena and J.A. Malpica, "An automatic method for road extraction in rural and semi-urban areas starting from high resolution satellite imagery," Pattern Recognit. Lett. (Netherlands), vol. 26, no. 9, pp. 1201 - 20, 2005.

[4] Darlis Herumurti, Keiichi Uchimura, Gou Koutaki, and Takumi Uemura, "Urban road extraction based on hough transform and region growing," in 19th Korea-Japan Joint Workshop on Frontiers of Computer Vision, (FCV), Jan 2013, pp. 220-224.

[5] A.K. Shackelford and C.H. Davis, "Urban road network extraction from high-resolution multispectral data," in 2nd GRSS/ISPRS Joint Workshop on Remote Sensing and Data Fusion over Urban Areas, 2003., May 2003, pp. 142-146.

[6] Mohamed Naouai, Atef Hamouda, Aroua Akkari, and Christiane Weber, "New approach for road extraction from high resolution remotely sensed images using the quaternionic wavelet," in Pattern Recognition and Image Analysis, vol. 6669, pp. 452-459. Springer Berlin Heidelberg, 2011.

[7] E. Salari and Y. Zhu, "A road extraction method using beamlet transform," in IEEE International Conference on Electro/Information Technology (EIT), 2012, May 2012, pp. 1-4.

[8] DavidL. Donoho and Xiaoming Huo, "Beamlets and multiscale image analysis," in Multiscale and Multiresolution Methods, vol. 20, pp. 149-196. Springer Berlin Heidelberg, 2002.

[9] J Canny, "A computational approach to edge detection," IEEE Trans. Pattern Anal. Mach. Intell., vol. 8, no. 6, pp. 679-698, June 1986. 